\title{
PART TILTING IN CAPILLARY-BASED SELF-ASSEMBLY: MODELING AND CORRECTION METHODS
}

\author{
Shaghayegh Abbasi ${ }^{1}$, Andrew X. Zhou', Rajashree Baskaran ${ }^{1,3}$, and Karl F. Böhringer ${ }^{1}$ \\ ${ }^{1}$ Dept. of Electrical Engineering, ${ }^{2}$ Dept. of Bioengineering, University of Washington, WA, USA \\ ${ }^{3}$ Intel Corporation, Chandler, AZ, USA
}

\begin{abstract}
We present a model and experimental results on tilt angle of microparts in capillary-driven self-assembly. The assembly is carried out in an aqueous environment, using a heat curable adhesive for part-substrate lubrication and mechanical bonding. Silicon parts and substrate have matching hydrophobic binding sites, which drive the assembly by surface energy minimization. Force balance analysis of an assembled part leads to a model describing the dependence of tilt angle on assembly parameters such as adhesive volume and water-adhesive interfacial tension. The effect of adhesive volume on tilt angle is investigated experimentally. Tilt correction of the assembled parts is achieved by providing external energy to the system via vertical vibration.
\end{abstract}

\section{INTRODUCTION}

Interest in the assembly of wafer-scale 3-dimensional microelectronics with high interconnect density has significantly increased in recent years due to the need for smaller sizes and higher speeds [1]. The electronic parts are usually very thin to optimize the form factor, hence they are hard to handle with pick and place assembly robots. In addition, a very high alignment accuracy $(<1 \mu \mathrm{m})$ is needed due to the small size and high density of interconnects, which requires a very expensive pick and place assembly setup.

One good candidate to accomplish these assemblies is capillary-based self-assembly, mainly because of its parallel nature and self-alignment capability [2]. The assembly is performed in two steps: electronic parts are first arranged on an assembly template using capillary forces acting on their back sides, and then they are transferred and bonded to the final substrate (Fig.1). In the first step, an adhesive is usually used between the parts and assembly template, which pulls in and precisely aligns the part due to surface energy minimization. However, part tilting interferes with sub-micron alignment accuracy, which is critical for high interconnect density. Investigation and correction of part tilt is essential for assembly processes mainly because tilt interferes with alignment accuracy, and also tilt is more difficult to control than lateral or rotational misalignment [3]. Eliminating tilt is also very important for optical applications such as the assembly of micromirrors. Previous studies on tilt have given a critical volume of the adhesive, below which tilt does not happen [4]. Here, a model of tilt angle as a function of assembly parameters is developed, which makes it possible to minimize the tilt angle by controlling parameters such as adhesive volume and water-adhesive interfacial tension. In addition, a method of tilt correction is demonstrated, which is especially useful when assembly parameters are determined by boundary conditions, or are hard to control.

\section{DESIGN AND FABRICATION}

\section{Design}

In order to use capillary-driven self-assembly for applications like wafer-scale 3-dimensional microelectronics with high interconnect density, high part lateral size to thickness ratio is needed. Consequently, we use $5 \times 5 \times 0.1 \mathrm{~mm}^{3}$ silicon parts. Since very high alignment accuracy $(<1 \mu \mathrm{m})$ is needed, we cannot use the diced edge of the part for aligning; instead, lithographically defined binding sites are used because lithography is much more accurate than dicing. The binding sites are rectangular, which allows for asymmetry and thus more flexibility in the circuitry design. Simulations have shown that the length to width ratio of rectangular binding sites needs to be more than 1.3:1 to get right assembly orientations [5]. Consequently, the binding site size is chosen as $3.65 \times 4.9 \mathrm{~mm}^{2}$.

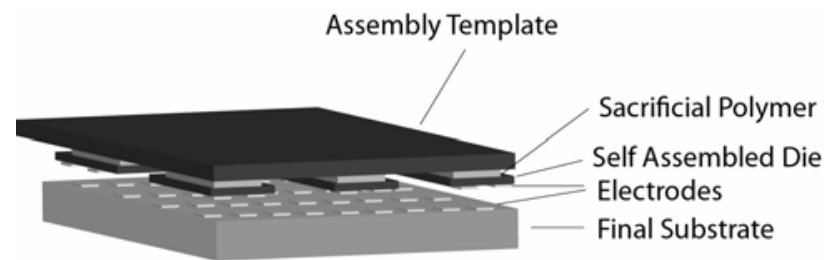

Figure 1: Wafer-scale assembly of electronic parts: First, parts with circuitry and interconnect pads are assembled on the assembly template. Then they are flipped on the final substrate, which has complementary circuitry and same-size interconnect pads. The interconnect pads on parts and substrate are connected using solder or metal-metal bonding.

\section{Fabrication}

The assembly template is fabricated on a 4" silicon wafer. The fabrication process is shown in Fig.2. The binding sites are defined by spin coating and lithographically patterning positive photoresist AZ4620, and then evaporating $\mathrm{Cr}$ and $\mathrm{Au}$ with thicknesses $100 \AA$ and $1000 \AA$, respectively. The $\mathrm{Cr}$ acts as an adhesion layer for Au. Next, a lift-off process is done by soaking the wafer in acetone overnight to define the final shape of the binding sites. In order to make the gold binding sites more hydrophobic, the wafer is plasma cleaned with $\mathrm{O}_{2}$, and then soaked in a solution of $1 \mathrm{mM}$ dodecanethiol in ethanol overnight. In this step a self-assembled monolayer (SAM) of thiol molecules selectively attaches to the gold binding sites and make them more hydrophobic [6]. The 
average contact angle of water on SAM-coated gold is measured as $110^{\circ}$.

Fabrication of dummy parts is achieved by lithography, evaporation and lift-off steps similar to the assembly template explained above. SAM coating is not done for the parts since untreated $\mathrm{Au}$ surfaces have a contact angle of $70^{\circ}$ after exposure to lab atmosphere [7], which is sufficient to ensure assembly. After lift-off, a grinding process from the back side of the wafer thins the silicon wafer to $100 \mu \mathrm{m}$, and then the wafer is diced into individual parts. Here we use silicon parts without any interconnect pads, and only study the self-assembly and improvement of alignment accuracy.

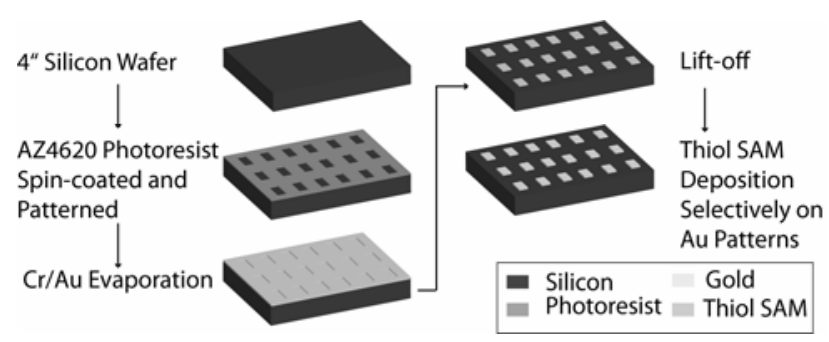

Figure 2: Fabrication process of substrate and parts.

\section{SELF-ASSEMBLY PROCESS}

The general process for part assembly is shown in Fig.3. The lubricant used for assembly is a hydrophobic heat curable adhesive composed of 97 wt.\% triethyleneglycol dimethacrylate as a monomer, and 3 wt.\% benzoyl peroxide as the thermal initiator [6]. First, the assembly template is immersed in the polymer and pulled out into the water (Fig.3 (a)). As a result of surface energy minimization and hydrophobicity of adhesive and substrate binding sites, the adhesive selectively covers the hydrophobic binding sites. In the next step, parts are introduced to the template, and due to surface energy minimization, they attach to the adhesive-coated binding sites (Fig.3 (b)). After assembly completion, the adhesive is cured by heating the water to $70{ }^{\circ} \mathrm{C}$ for 2 hours, and mechanical bonding is achieved.

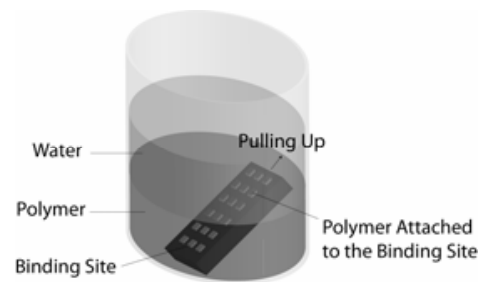

(a)

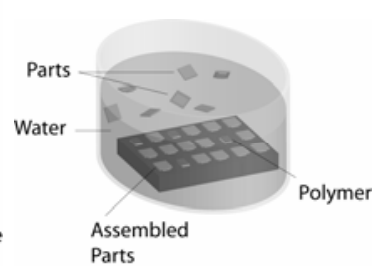

(b)
Figure 3: (a) Selective coating of sites with heat curable hydrophobic adhesive. (b) Delivery of parts in water medium that results in formation of a capillary bridge between the hydrophobic sites of the parts and the substrate. The adhesive is then heat cured for mechanical bonding.

\section{MODELING AND EXPERIMENTS}

\section{Modeling}

The tilt angle of the assembled part is modeled as a function of assembly parameters, using a force balance analysis. It is assumed in our calculations that one edge of the tilted part is always touching the substrate, which is true for more than $90 \%$ of the parts according to our experiments. The main forces on the part are gravity force and capillary force, as shown in Fig.4 (a). The capillary force can be calculated according to the Young-Laplace equation as follows:

$F_{c}=\Delta P A=\gamma_{A W}\left(\frac{1}{R_{1}}+\frac{1}{R_{2}}\right) A$

where $F_{c}$ is the capillary force, $\Delta P$ is the pressure difference between adhesive and water, $\gamma_{A W}$ is the adhesive-water interfacial tension, $A$ is the binding site area, and $R_{1}$ and $R_{2}$ are the radii of curvature as shown in Fig.4. $R_{1}$ describes the highest curvature, which is at the bulge of the adhesive along the vertical direction. $R_{2}$ is the radius of the waist of the adhesive meniscus between part and substrate. $\gamma_{A W}=15.2$ $\mathrm{mN} / \mathrm{m}$, which is measured using the pending drop method in a goniometer. The area $A$ is given using the binding site geometry. $R_{2}=L / 2$, assuming that the adhesive drop is approximately a cylinder (Fig.4 (a)), where $L$ is the length of the binding site. Thus the only unknown parameter in Eq. 1 is $R_{1}$, which will be calculated using force balance.

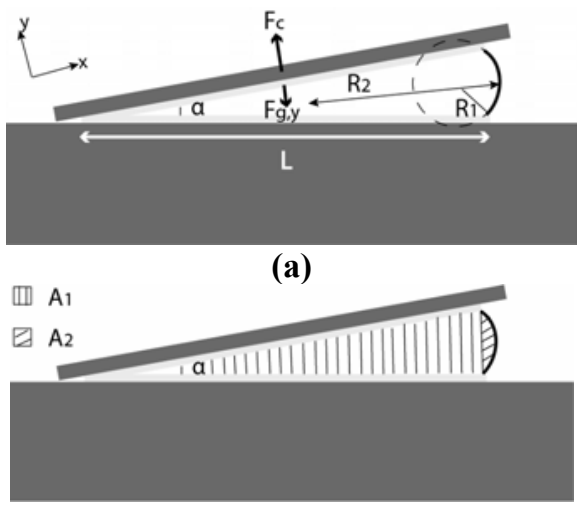

(b)

Figure 4: (a) Forces acting on the part. Force balance is used to calculate the relationship between the tilt angle and adhesive volume. (b) Cross-sectional areas $A_{1}$ and $A_{2}$ are added up to calculate the adhesive volume as a function of assembly parameters.

The gravity force component in $F_{c}$ direction, which is the important component for us since we are doing a force balance calculation, is shown in the following equation:

$F_{g, y}=m g \cos (\alpha)$

where $F_{g, y}$ is the gravity force component perpendicular to the part (in $F_{c}$ direction), $m$ is the part mass, $g$ is gravitational acceleration, and $\alpha$ is the tilt angle of the part.

If the total force on the part becomes zero as it is tilted, it is in equilibrium and will not level out. Consequently, the 
equilibrium tilt angle $\alpha$ can be calculated by solving the following equation:

$F_{t o t}=F_{c}-F_{g, y}=0$

Substituting Eq. 1 and Eq. 2 in Eq.3, $R_{1}$ is deduced as a function of $\alpha$ and assembly parameters:

$R_{1}=\left(\frac{\operatorname{t\rho g} \cos (\alpha)}{\gamma_{A W}}-\frac{1}{R_{2}}\right)^{-1}$

where $t$ and $\rho$ are the part thickness and density, respectively. Next, $R_{1}$ is used to calculate area $A_{2}$ in Fig.4 (b), which is then used in the equation $V=\left(A_{1}+A_{2}\right) W$ to calculate adhesive volume. Here $V$ is the adhesive volume, $W$ is the width of the binding site, and $A_{1}$ and $A_{2}$ are the areas shown in Fig.4 (b). The following relation is derived between the adhesive volume and part tilt angle $\alpha$ :

$V_{\alpha}=W L^{2} \sin (\alpha / 2) \cos (\alpha / 2)+$

$+W\left(R_{1}^{2} \beta-L \sin (\alpha / 2) R_{1} \cos (\beta)\right)$

where $R_{1}$ is calculated in Eq.4, $\beta=\sin ^{-1}\left(L \sin (\alpha / 2) / R_{1}\right), W$ and $L$ are binding site width and length, and $\alpha$ is the tilt angle. Eq.5 is useful especially for applications where there is a limit for tilt angle. For example, if the tilt angle needs to be less than $\alpha_{\text {limit }}$, this angle can be substituted in Eq.5, and the maximum allowed volume can be calculated. For small tilt angles $\left(<10^{\circ}\right)$, Eq.5 can be simplified to the following equation:

$V_{\alpha}=W L^{2} \sin (\alpha / 2)$

The adhesive contact angle in water does not appear in the equations, because it varies as the adhesive is pinned to the edges of the binding site, and can have different values depending on volume.

\section{Experiments}

Assembly experiments are done to evaluate the model. The assembly template is placed in water, and a micropipettor is used to deposit controlled adhesive volumes on the binding sites. In the next step, the parts are introduced and assembled to the substrate. The adhesive is then heat cured, and the template with assembled parts is dried carefully. Tilt angle of the parts is measured using optical photography. The volume range of adhesive in the experiments is from $3 \mu \mathrm{L}$ to $17 \mu \mathrm{L}$, with $2 \mu \mathrm{L}$ addition steps. At least 5 experiments are done and averaged for each volume. Fig. 5 shows the comparison between the model (Eq.5) and the experimental results. One possible reason for model-experiment disparity is the assumption of one part edge touching the substrate in the modeling, which is not always correct.

As can be seen in the plot, the tilt angle increases by adhesive volume increment, both in the model and in the experimental results. Consequently, the tilt angle can be minimized by minimizing the adhesive volume. However, this is not always a possible solution since the adhesive is usually deposited on the binding sites by dip-coating (Fig.3 (a)), where the control range of volume-determining parameters is very limited. These parameters include dipping velocity, adhesive viscosity, and adhesive-water interfacial tension. In addition, the adhesive volume needs to be higher than a minimum value so that it covers the binding site completely. In the next section, other methods are proposed to minimize and correct the part tilt, which do not need control over assembly parameters.

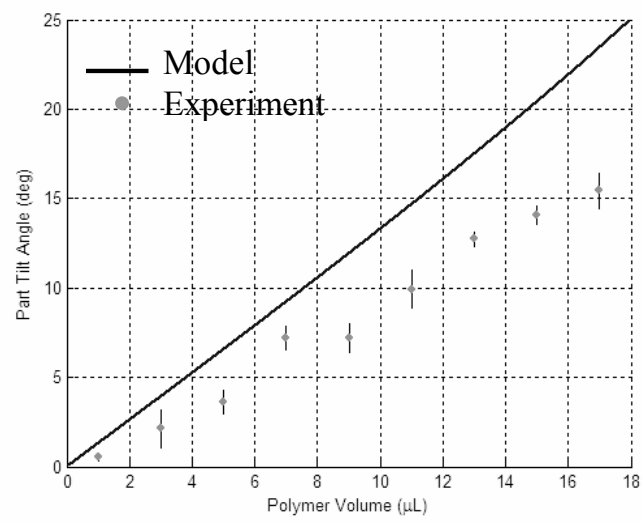

Figure 5: Part tilt angle as a function of polymer volume, model and experimental results. Error bars show standard deviation for 5 experiments. The assumption of part edge contact is a possible reason for model-experiment disparity.

\section{CORRECTION METHODS}

During capillary-driven self-assembly, the direction at which parts approach the binding sites is random. The chance of parts approaching vertically with good alignment is low. Parts mainly approach from the side, which makes the polymer distribution nonuniform (Fig.6); hence the part reaches a tilted equilibrium position (local energy minimum), i.e., although the tilted state is equilibrium, it is caused only as a result of geometric constraints, and is not the global energy minimum of the system.

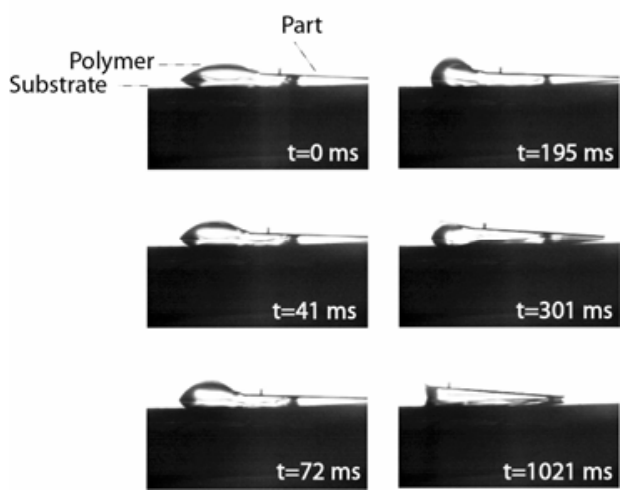

Figure 6: Part assembled from right side, it tilts as assembly proceeds.

Surface Evolver [8] simulations are used to find the global energy minimum of the system. The system is evolved for tilt angles from zero to the maximum angle for a constant adhesive volume, and energy of each state is plotted as a function of tilt angle. Simulation results for $V_{\text {adhesive }}=15 \mu \mathrm{L}$ are shown in Fig.7. The plot shows that the energy minimum is at 
tilt angle $\alpha=0^{\circ}$, which means the flat state. Consequently, by giving external energy to the system, the part edge can overcome stiction to the substrate, so the system can be conducted to its global energy minimum and parts can level out. It is worth mentioning that since stiction of part to the substrate is not taken into account in simulations, the energy barrier from the tilted state to the flat state does not appear in the simulation results.

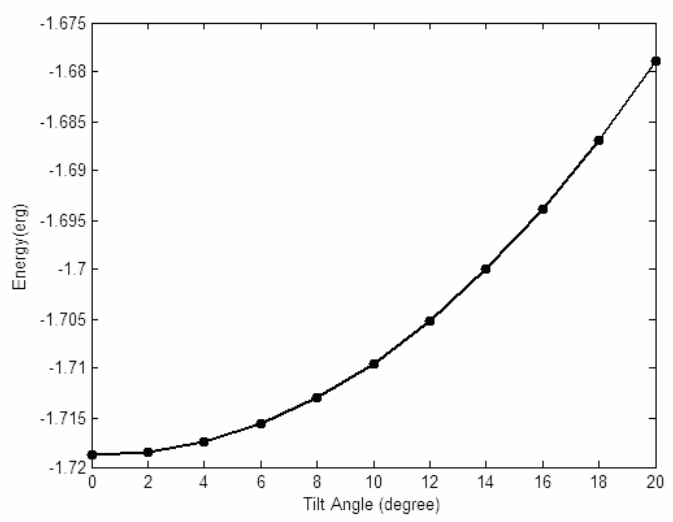

Figure 7: Part-adhesive-substrate system energy as a function of part tilt angle for $V_{\text {adhesive }}=15 \mu L$. The global energy minimum of the system is at the flat state.

Here vertical vibration is used as a method of external agitation. The template is attached firmly to the bottom of a glass container, which is then filled with low level water. Controlled adhesive volumes are deposited on binding sites using a micropipettor, followed by part assembly. Next, the container is glued to the stage of a speaker, connected to a signal generator with a $15 \mathrm{~Hz}, 9 \mathrm{~V}$ sinusoidal wave for 2 minutes. The tilt angle of the parts is measured using optical photography before and after vibration for different volumes. At least five experiments are done and averaged for each volume. The tilt correction can be clearly seen in Fig.8 (a) and (b), where an assembled part is shown before and after vibration, respectively. Fig.8 (c) shows a plot of part tilt angle before and after vibration for different adhesive volumes.

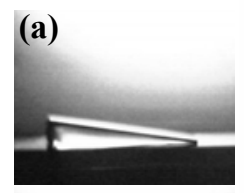

(b)

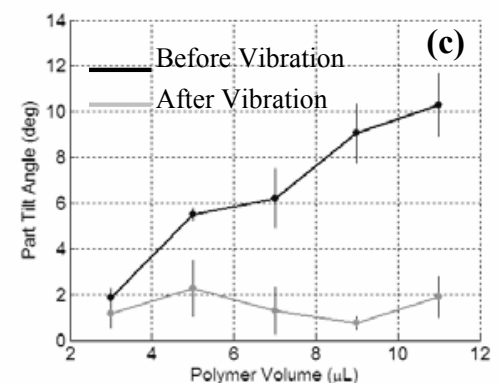

Figure 8: (a) Tilted part after assembly. (b) Tilt is corrected after vibration. (c) Tilt angle of assembled parts before and after vibration. Each experiment is done at least 5 times, and error bars show standard deviation.

The vibration helps with tilt correction in two ways: first is the kinetic energy it gives to the system as a result of movement, and second is the energy of water surface waves, which are formed in close contact with the parts and substrate because of low water level in the container. According to the experimental results, after giving external energy to the system through vibration, the tilt is corrected to around or less than $2^{\circ}$, regardless of the initial tilt angle. This confirms that here the tilt is a result of geometric constraints, and the global energy minimum for all volumes, which is maintained after giving external energy, is the flat state.

\section{CONCLUSION}

This work makes control and minimization of part tilt in capillary-based self-assembly possible by modeling the tilt angle as a function of assembly parameters. Since the exact control of assembly parameters is not always possible due to boundary conditions, a tilt correction method is demonstrated. The key point in the tilt correction is giving external energy to the system so that it reaches its global energy minimum, which is the flat state. Here vibration is used as the external agitation. Future work will be aimed on investigation of scaling effects on tilt angle and correction methods.

\section{ACKNOWLEDGEMENTS}

This work was supported by research grants from Intel Corporation. A. Z. was supported by UWEB REU program at the University of Washington. The staff of the Microfabrication Laboratory at the Washington Technology Center provided help in cleanroom microfabrication processes.

\section{REFERENCES}

[1] S. Savastiouk, O. Siniaguine, and E. Korczynski, "3D Stacked Wafer-Level Packaging”, Advanced Packaging, March 2000, pp. 28-34.

[2] U. Srinivasan, D. Liepmann, and R.T. Howe, "Microstructure to Substrate Self-Assembly Using Capillary Forces", J. Microelectromech. Syst., Vol.10, No.1, pp. 17-24, 2001.

[3] U. Srinivasan, "Fluidic Self-Assembly of Microfabricated Parts to Substrates Using Capillary Forces", Ph.D. Dissertation, 2001.

[4] K.L. Scott, R.T. Howe, and C.J. Radke, "Model for Micropart Planarization in Capillary-Based Microassembly", The 12th International Conference an Solid State Sensors, Actuators and Microsystems, Boston, June 8-12, pp. 1319-1322, 2003.

[5] J. Fang, K. Wang, and K.F. Böhringer, "Self-Assembly of PZT Actuators for Micro Pumps with High Process Repeatability", J. Microelectromech. Syst., Vol.15, No.4, pp. 871-878, 2006.

[6] X. Xiong, Y. Hanein, J. Fang, Y. Wang, W. Wang, D.T. Schwartz, and K.F. Böhringer, "Controlled Multibatch Self-Assembly of Microdevices", J. Microelectromech. Syst., Vol.12, No.2, pp. 117-127, 2003.

[7] C. D. Bain, E. B. Troughton, Y. T. Tao, J. Evall, G. M. Whitesides, and R. G. Nuzzo, "Formation of monolayer films by the spontaneous assembly of organic thiols from solution onto gold," J. Amer. Chem. Soc., vol. 111, pp. 321-335, 1989.

[8] K. Brakke, Surface Evolver website, www.geom.umn.edu/software/download/evolver.html. 